\title{
Targeting 1-Deoxy-D-xylulose-5-Phosphate Synthase (DXS) in Plasmodium Species for New Generation of Antimalarial
}

\section{Sumit Handa*}

Department of Chemistry and Biochemistry, University of California, San Diego, California, USA

Malaria is one of the most dreadful diseases on the planet earth and nearly half of the world population is at a risk of this infection. Because of the advancement in medication and therapeutics, malaria related death toll has seen a 48\% drop from estimated 839,000 in 2000 to 438,000 in 2015 [1,2]. Most of this life-threatening disease causality is in tropical climate of Sub-Saharan Africa (90\%) and South-East Asia $(7.5 \%)$ and because of the weak infrastructure in some countries the challenge to fight this disease has been an uphill battle. Multiplied with the parasitic, intra-cellular life cycle of the Plasmodium species $(P$. falciparum, $P$. vivax, $P$. malariae and $P$. ovale) makes it even harder to develop vaccine. Current antimalarial medications such as artemisinin, chloroquine, sulfonamides, pyrimethamine, etc. are failing in treatment of malaria because of increasing drug-resistance by Plasmodium species.

This drug resistance has been recurring problem to treat various parasitic diseases (caused by Trypanosoma brucei, Toxoplasma gondii, Leishmania species) and bacterial infectious diseases (caused by Staphylococcus aureus, Group A Streptococcus, Clostridium difficile, Neisseria gonorrhoeae) as well. The only solution to these arduous problems is through development of new druggable targets. The whole genome sequence of $P$. falciparum has provided information about the vital metabolic pathways used for survival and resistance; and how these can be targeted to develop new generation of antimalarial [3]. Some of the most relevant biological target in Plasmodium that has been identified and localized, such as 2-C-methyl-D-erythritol 4-phosphate (MEP) pathway, type II fatty acid synthesis, iron sulfur cluster assembly, fragmented heme synthesis [3], and complete absence in the mammalian host, makes them an appealing target for drug discovery. All these pathways have been localized into apicoplast of Plasmodium, an essential organelle that is homologous to plants and algae plastids [4,5]. All the proteins involved in the above mentioned pathways are nuclear encoded and transported to apicoplast through a two steps process with the help of signal and transit peptide [4], making this organelle indispensable for Plasmodium survival. Some bacteria also use this mevalonate-independent pathway for isoprenoid synthesis, thus making this pathway an attractive target for antibiotics development.

Various proteins in MEP pathway have recently been targeted for antimicrobial drug development. 1-Deoxy-D-xylulose-5-Phosphate Synthase (DXS) the rate-limiting enzyme of MEP pathway, which catalyze the first step in MEP pathway through biosynthesis of 1-deoxyD-xylulose-5-phosphate (DXP), by condensation of pyruvate and glyceraldehyde 3-phosphate (GAP) is example of an appealing target [6]. DXS belongs to thiamine diphosphate (TDP) dependent enzyme and share a high homology with mammalian pyruvate dehydrogenase, and transketolase [7]. But DXS display some distinct structural [7] and mechanistic features [8,9] which sets it apart from other TDP-dependent enzymes, making this as an attractive target for drug development.

Some of the very first study reported was on structure-activity target development against DXS of Mycobacterium tuberculosis, using known transketolase inhibitor [10]. This study reported an inhibitor of M. tuberculosis DXS with and $\mathrm{IC}_{50}$ of $10.6 \mu \mathrm{M}$. After this report several other findings were also published, where acetylphosphonate and hydroxybenzaldoximes derivatives, mimicking the active-site pocket of DXS were synthesized and shown a promising inhibition of $E$. coli DXS at $\mathrm{IC}_{50}$ value for some derivatives just below $10 \mu \mathrm{M}[11,12]$. Antimicrobial $\mathrm{N}$-(2-chlorobenzyl)-substituted hydroxamate (hydrolysis product of ketoclomazone) shows inhibition for Haemophilus influenza DXS, even at lower $\mathrm{IC}_{50}$ value of $1.0 \mu \mathrm{M}$ [13]. Recent report on the development of DXS from $P$. falciparum and $P$. vivax using an overexpression system in E. coli, has open an attractive target for new generation of antimalarial discovery $[14,15]$ as well. DXS from both the Plasmodium species are inhibited by $\beta$-Fluoropyruvate (F-Pyr) and methylacetylphosphonate (MAP), though a competitive inhibition with respect to pyruvate and noncompetitive against GAP [15]. A homology model of P. vivax and other Plasmodium species (P. falciparum, P. berghei) DXS has also been reported recently, and has been validated for virtual screening of target using NCI diversity set [16]. All the inhibitors developed so far against bacterial DXS have still to be tested against Plasmodium DXS to check their efficacy and then come up with a moiety to design a better drug candidate.

Current challenges with the development of selective and new generation of antimalarial have been hindered by the lack of new targets. Since DXS is involved in the first rate-limiting step in the MEP pathway and also shows feedback inhibition by the end product of MEP pathway [9], thereby making DXS a convincing drug development target in the field of malaria therapeutics.

\section{References}

1. (2015) World Health Organization, World Malaria Report 2015.

2. Valipour M, Singh VP (2016) Global experiences on wastewater irrigation Challenges and prospects. Balanced urban development: Options and strategies for liveable cities, eds Maheshwari B, Singh PV, Thoradeniya B (Springer International Publishing, Cham), pp: 289-327.

3. Gardner MJ (2002) Genome sequence of the human malaria parasite Plasmodium falciparum. Nature 419: 498-511.

4. McFadden GI, Reith ME, Munholland J, Lang-Unnasch N (1996) Plastid in human parasites. Nature 381:482.

5. Wiesner J, Seeber F (2005) The plastid-derived organelle of protozoan human parasites as a target of established and emerging drugs. Expert Opin Ther Targets 9: 23-44

6. Estevez JM, Cantero A, Reindl A, Reichler S, Leon P (2001) 1-Deoxy-Dxylulose-5-phosphate synthase, a limiting enzyme for plastidic isoprenoid biosynthesis in plants. J Biol Chem 276: 22901-22909.

7. Xiang S, Usunow G, Lange G, Busch M, Tong L (2007) Crystal structure of 1-deoxy-D-xylulose 5-phosphate synthase, a crucial enzyme for isoprenoids biosynthesis. J Biol Chem 282: 2676-2682.

8. White JK, Handa S, Vankayala SL, Merkler DJ, Woodcock HL (2016) Thiamin diphosphate activation in 1-Deoxy-d-xylulose 5-Phosphate Synthase: Insights

*Corresponding author: Sumit Handa, Department of Chemistry and Biochemistry, University of California, San Diego, California, USA, Tel: 0018134204483 E-mail: suhanda@ucsd.edu

Received September 20, 2016; Accepted October 03, 2016; Published October 10,2016

Citation: Handa S (2016) Targeting 1-Deoxy-D-xylulose-5-Phosphate Synthase (DXS) in Plasmodium Species for New Generation of Antimalarial. Mol Biol 5: 175 doi:10.4172/2168-9547.1000175

Copyright: (c) 2016 Handa S. This is an open-access article distributed under the terms of the Creative Commons Attribution License, which permits unrestricted use, distribution, and reproduction in any medium, provided the original author and source are credited. 
Citation: Handa S (2016) Targeting 1-Deoxy-D-xylulose-5-Phosphate Synthase (DXS) in Plasmodium Species for New Generation of Antimalarial. Mol Biol 5: 175. doi:10.4172/2168-9547.1000175

into the mechanism and underlying intermolecular interactions. The Journal of Physical Chemistry B.

9. Banerjee A (2013) Feedback inhibition of deoxy-D-xylulose-5-phosphate synthase regulates the methylerythritol 4-phosphate pathway. J Biol Chem 288: 16926-16936.

10. Mao J (2008) Structure-activity relationships of compounds targeting Mycobacterium tuberculosis 1-deoxy-D-xylulose 5-phosphate synthase. Bioorg Med Chem Lett 18: 5320-5323.

11. Bartee D, Morris F, Al-Khouja A, Freel Meyers CL (2015) Hydroxybenzaldoximes Are D-GAP-competitive inhibitors of E. coli 1-Deoxy-D-Xylulose-5-Phosphate Synthase. Chembiochem 16: 1771-1781.

12. Smith JM, Vierling RJ, Meyers CF (2012) Selective inhibition of E. coli 1-deoxyD-xylulose-5-phosphate synthase by acetylphosphonates. Medchemcomm 3:65-67.
13. Hayashi D, Kato N, Kuzuyama T, Sato Y, Ohkanda J (2013) Antimicrobial N-(2 chlorobenzyl)-substituted hydroxamate is an inhibitor of 1-deoxy-d-xylulose 5-phosphate synthase. Chemical Communications 49: 5535-5537.

14. Handa S (2013) Production of recombinant 1-deoxy-d-xylulose-5-phosphate synthase from Plasmodium vivax in Escherichia coli. FEBS Open Bio 3: $124-$ 129.

15. Battistini MR, Shoji C, Handa S, Breydo L, Merkler DJ (2016) Mechanistic binding insights for 1-deoxy-D-Xylulose-5-Phosphate synthase, the enzyme catalyzing the first reaction of isoprenoid biosynthesis in the malaria-causing protists, Plasmodium falciparum and Plasmodium vivax. Protein Expr Purif 120: 16-27.

16. Ramamoorthy D HS, Merkler DJ, Guida WC (2014) Plasmodium vivax 1-deoxyD-xylulose-5-phosphate synthase: Homology modeling, domain swapping and virtual screening. J Data Mining Genomics Proteomics S1: 2153. 